\title{
An Exploration of HCI Design Features and Usability Techniques in Gaming
}

\author{
Venkata Krishna Mahesh Kumar Kondraju \\ School of Computer and Information Science / University of South Australia, Australia
}

\begin{abstract}
This Conference paper explores the human computer interaction design features relating to gaming industry. The HCI user design interface development and HCI Usability expectations are discussed in this paper. HCI design features of Simple Sudoku and Contemporary HCI Expectations are also discussed. And suggestions are given to develop the Simple Sudoku as per HCI standards and expectations.
\end{abstract}

Keywords: Brain Computer Interface, Gestures Recognition, Hand Gestures in 3D, Pattern Language Management Tool, Real Time Strategy Games

\section{Introduction}

HUMAN COMPUTER INTERACTION in this world is currently working with software industries such as gaming industry to implement $\mathrm{HCI}$ user design features and usability techniques to develop usable and interactive games [1], [14]. Industry Practices expect design decisions, user interface designs and 'usability testing to be more collaborative' such that whole industry will gain and can move forward with confidence [8]. So with this HCI's importance or impact on software industry has been identified [1], [14].

Though few years back or few decades back HCI and games were not working together as they would fall under different set of development strategy's, as games were more practical based with more graphical user interface designing with less user centric design features as they were designed according to developers strategy $\&$ game play. While Human computer interaction was more of theoretical based architecture which studies the usability and design features of the games [1].

\section{HCI Usability, Design Process, 3d Real Time Games With Gestures}

\subsection{Routes for Designing Games with Usability}

HCI usability was Key to modify the interface and to redesign the features of computer games. But uniting $\mathrm{HCI}$ and computer games lead to few architectural changes and they are as follows:

2.1.1 Development on keen watching.

2.1.2 Effective gaming with effortless community.

Customizing according to users perspective which is also known as deep customizing, which gives power to the users to set $\&$ play the game according to their one's own desire. For example setting the stages or levels of game play by skipping previous stages or even changing the graphical user interface by changing backgrounds, colors, use of tools, screen layout, playing game in a window screen or full screen game play etc.

Usability levels of games are redefined with visual experience which relies on design features.

HCI technique Fluid System is helpful in designing a game which makes sure that any other events such as system messages or error messages or focusing to other application which resume's from a sleep doesn't disturb the game of play. This technique needs to be adopted for keeping alive gamers interests, to have a continuous game play without interruptions [1].

\subsection{Real time 3D games}

The gaming industry with the interaction of HCI is developing new standards such as user visual experience for the games by designing screen layout, developing more realistic 3 dimensional images, true graphics, navigation, selection of commands or buttons used in game play [2].

Now a days as Real time games are concentrating more on user experience and for this user experience industry is developing, modifying or redesigning the interfaces of the game with the real time strategies in role play of the game with the introduction 3 dimensional design interfaces [2].

Certain design principles were followed while developing a usable but at the same time also an enjoyable interface in real time gaming. In real time 3D games user experience is important, so a practical interpretation of user ideas \& one's desires are considered while developing a 3D interface. Visual techniques 
are used to dramatize the events in the game (such as adventure games) to get a new feel with the interface. So visual techniques are adopted and play a very important role in designing a $3 \mathrm{~d}$ real time games and have become the primary goal for the developers [9].

3D games are scenario based games with 3 dimensional environments due to its typical behavior a behavioral model is developed which is known as GSOMS. GSOMS model stands for Goal Scenario Operator Methods Selection Rule. This model is used as a base to develop a 3D game [2].

The rules or guidelines to follow in developing a 3D user interface in a Real time 3D game by using a scenario based behavior model GSOMS are:

\section{Check Figure 2.2.1 Command \& Conquer 3D Interface for Real-time Strategy Games [2]}

2.2.1 The architecture of the game is Constructed in such way that it should have a compact $\&$ clear screen layout with a main window in it with Picture type frames (B-frames, I-frames, P-frames) with good video acceleration support( hardware support). In short interface needs to meet the standards set by GSOMS.

2.2.2 Enhancement of audio for 3D gaming purposes known as 3D audio.

2.2.3 Emphasis on background, screens, cursors and picture frames, which resembles visual experience.

2.2.4 Provision for instant feedback which leads to an efficient development [2].

\subsection{Hand Gestures in 3D Real Time Gaming Using HCI}

Human computer interaction's introduction of hand gestures generates interest in the industry, while hand gestures are used to 'play a natural and intuitive communicational mode of all human dialogs' [3].

In this figure 2.3 [3] shows that hand gestures are used as commands to control the games instead of keyboards or mouse. In order use these gestures as commands for the computer games, a software is used to detect the 3D positions of the gestures such as hand gestures, finger pointing gestures of thumb \& index finger and 3D position of user's head. For Utilization of these 3D positions a visual gesture interface is developed to interact with the game. This visual gesture interaction between human operator \& computer environment has been enabled with the help of a theory known as sign language recognition along with kalman filter based prediction [3], [7] and [20].

Researchers consider Hand gestures further leading to the introduction of pointing gestures is 'one of the most natural \& intuitive HCI approach' [7].

Combination of these techniques in HCI leads to better user interface design. There are few concepts or approaches developed for betterment of user interfaces they are:

\section{Intelligent user interfaces}

User interface design is achieved by adapting to the concepts such as 'intelligent user interfaces which provides the knowledge of background information for designing [4].

\section{Adaptive user interfaces}

It is the interface developed from collective feedback received from users or multiple gamers, this feedback is used to utilize the knowledge of users to design or configure the interface [4].

\section{User interface management systems}

In order to develop the application as a whole designing needs to be separated from the application by following the concept of DCT (Design, Coding, Testing) this separation of designing from application is done under user interface management systems (UIMS) [4].

Multimedia user interfaces

These are used to provide rich environment for user while playing the games [4].

\subsection{Gestures Recognition with WII Controller}

Latest current technology which is also triggering gaming industry is Wii controller which is also called as Wii remote. This technology is most widely used in game consoles such as Nintendo, Xbox360 etc. This HCI technology is a remote optical sensor technology with which a game console can be provoked with the input commands. With the introduction of this technology there is a regulatory change in interface design for the games [10].

\subsection{Implementation of a 3-Dimensional Game Using BCI}

$\mathrm{BCI}$ (Brain wave computer interaction) which is a part of $\mathrm{HCI}$ and is the one of the research areas on which everyone are concentrating nowadays [5]. 
Brainwave computer interaction concentrates more on how the research has been conducted for the implementation of HCI techniques. This research affects the user interface design and triggers a new interface with fresh new ideas [5].

BCI research spells out on pattern recognition mechanism which helps in adding flexibility to the interfaces [5].

Pattern recognition is built using Pattern Language Management Tool (PLMT). This PLMT is used to manage constructed languages in HCI, it is an automated tool searches for pattern's present in HCI language, and this also helps in adding patterns such as interface design patterns to the HCI languages and also helps in modifying existing interfaces [11].

BCI's research on new interface has amazed gamers and not only gamers but also gaming companies are surprised with the new interface coming up. BCI's new interface is a kind of interface where all important guidelines or research information from HCI are taken into consideration such as user centric designing, issues, visual experience etc [5].

Finally Brain wave computer interaction finally ousted with the interface developed which is more specific to user centric and the features of interface are as listed below:

2.5.1 Brain computer interface (BCI) is used along with special devices from BCI.

2.5.2 BCI interface is an unique and new concept where brain works as virtual game controllers rather than any strategies deployed or used by the developers.

2.5.3 BCI Devices are used to control the computer and the devices are controlled by brains of the user or gamer.

2.5.4 Thoughts of user are being transformed to the games virtually by the BCI devices.

2.5.5 This technology has created shivers in the gaming industry. In fact not only gaming industry but also doctors \& Scientists are worried with the introduction of this brain to game interface and its effects on gamers [5], [11], [21] and [22].

\subsection{GUI vs. User Centric Interfaces}

Graphical user interfaces which are very important in developing the games, but developed with no thought or respect for user's interest or ideas [6], [16]. But now a day's developers are keen to know the user's view point or perspective due to the complex nature of GUI's. This provoked for the introduction of user centric interfaces which resolves any complexity issues in interfaces. This shows that user centric interfaces are no different from GUI's but seem to be the superset of GUI's with few architectural changes in designing or developing the interfaces [6], [16].

\section{HCI Design Features Of Simple Sudoku}

A Simple Sudoku has a number based logic puzzle consisting of 9x9 grids which are further divided into $3 \times 3$ grids containing digits or numbers from 1-9 [12].

Initially Sudoku was known as 'Number place' but after gaining recognition it was renamed to Sudoku which is a Japanese word originated from Japan where 'Su' means number and 'Doku' means single [12].

Important Design features such as search space heuristics to arrive at problem initialization and neighborhood, which speeds up the solution with the implementation of Hill climbing algorithm for finding the unsolved puzzles or grids. Some more important features are hints to solve the puzzles, various complexity of playing levels, Multi language GUI which shows that the GUI used in the development of Simple Sudoku is an User Centric GUI [12], [15].

Algorithmic approaches used in the development of Sudoku are recommend from the research studies of HCI and is good enough to solve complex rated puzzles with reduced problem search place [12].

Algorithms are constructed in combination of logistics and a code written in a programming language for execution of pre-defined tasks, Along with this algorithm HCI standards implemented with heuristics search based approaches are designed for reducing the unsolved grids in the puzzle [12].

N-P complete puzzle of Sudoku which is solved using a stochastic search based algorithm which is also known as SA algorithm helps in discovering type of instances [13].

Various algorithmic approaches are applied throughout the development of Simple Sudoku in order to meet HCI expectations [13].

\section{CONTEMPORARY HCI EXPECTATIONS \\ 4.1 User Interface Meet Contemporary HCI Expectations \\ Simple Sudoku interface meets the contemporary HCI expectations as}


Simple Sudoku has followed the User Centric design interfaces such as:

4.1.1 Designing game according to one's logical thinking.

4.1.2 Easy, Standard, Hard, Extreme, Expert levels are introduced by keeping various gamers perspective in mind.

4.1.3 Hints are given to solve the puzzle.

4.1.4 Multi languages GUI support etc.

These design features are user centric as they are made available keeping in mind the interests of different users such as when an expert is playing he will choose the Expert mode, where as a starter can choose the easy mode [15].

\subsection{User Interface Does Not Meet Contemporary HCI expectations}

The contemporary HCI expectation for Simple Sudoku's interface which doesn't meet are:

4.2.1 3Dimensional interface.

4.2.2 Introduction of various new technologies to the interface.

\subsection{Suggestions for Improving Simple Sudoku HCI}

Well though as stated there are few Technologies lacking in the development of Simple Sudoku's interface such as

4.3.1 Introduction of 3 Dimensional interfaces.

4.3.2 User visual experience.

Still I think it's a well-crafted application or software to be played on normal computer platforms without expecting much support from hardware such as video acceleration, 3D support from the video card etc.

So final words on Simple Sudoku's interface is that though it lacks the introduction of few new technologies, it still meets all the Basic Contemporary HCI expectations such as User centric designing, use of different algorithmic approaches for solving puzzles of Simple Sudoku etc. This explains that Simple Sudoku's development has done as per HCI standards \& expectations.

\section{FIGURES AND TABLES}

Figure 2.2.1 Command \& Conquer 3D Interface for Real-time Strategy Games [2]

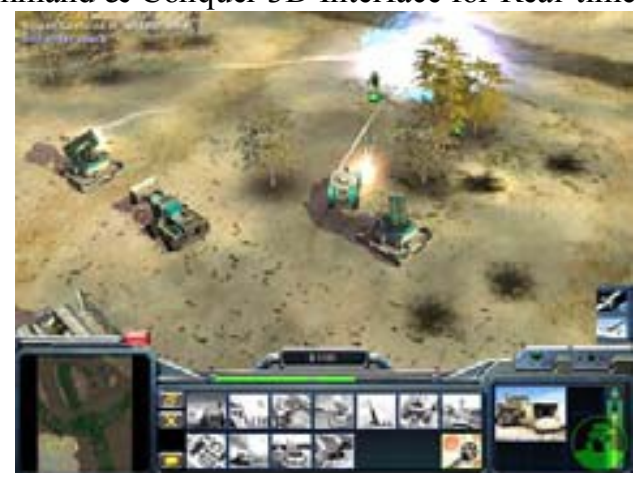

Figure 2.3 [3]

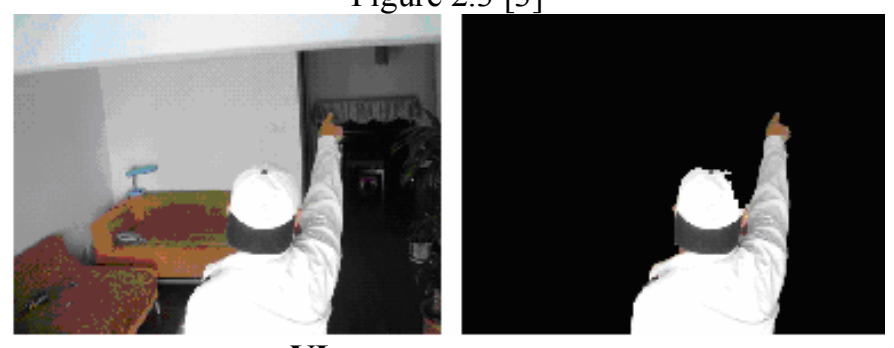

VI. CONCLUSION

HCI plays a major role in developing the games with good design interface and usability. Design interface in HCI is important whereas User Centric Design Interface is key to success. Many developers don't have the user's idea \& perspective in mind while developing a GUI. So it's important to develop an User Centric Design Interface. 


\section{REFERENCES}

[1] Jorgensen, A.H., 2004, 'Marrying HCI/Usability and Computer Games: A Preliminary Look', ACM International conference proceeding series, vol. 82, pp 393-396.

[2] Wenjun, H, Xiudong, B, 2006, 'HCI in real-time strategy games: a study of principals and guidelines for designing 3D user interface', Computer-Aided Industrial Design and Conceptual Design, pp. 1-6

[3] Guan, Y, Zheng, M, 2008, 'Real-time 3D pointing gesture recognition for natural HCI', Proceedings of the $7^{\mathrm{TH}}$ World Congress on Intelligent and Automation, pp. 2433-2436.

[4] Cudd, P.A., Oskouie, R, 1996, 'Combining HCI Techniques for Better User Interfacing', IEEE Colloquium on Interfaces - The Leading Edge (Digest No.1996/126), Vol.1, No. 11, pp. 1-9.

[5] Shim, B.S., Lee, S.W., Shin, J.H., 2007, 'Implementation of a 3-Dimensional Game for developing balanced Brainwave', Fifth International Conference on Software Engineering Research, Management and Applications, pp. 751-758.

[6] Polack-Wahl, J.A., 2007, 'Teaching HCI in Software Engineering', 34th ASEE/IEEE Frontiers in Education Conference, Vol. 2, pp $1-6$.

[7] Yi, B, Harris, F.C., Wang, L, Yan, Y, 2005, 'Real-Time Natural Hand Gestures', IEEE Computing In Science \& Engineering, Vol. 7, No. 3, pp. 92-96.

[8] Milewski, A.E., 2004, 'Software Engineers And HCI Practitioners Learning To Work Together: A Preliminary Look At Expectations', Proceedings of the 17th Conference on Software Engineering Education and Training, pp. 45-49.

[9] Grundy, C, Blake, A, 2007, 'Vis-able: Using Visual Communication Techniques to Improve Usability, Proceedings of the ITI 2007 29th Int. Conf. on Information Technology Interfaces, pp. 239-244.

[10] Schlomer, T, Poppinga, B, Henze, N, Boll, S, 2007, 'Gesture Recognition with A Wii Controller, Proceedings of the 2nd International Conference on Tangible and Embedded Interaction, pp. 11-14.

[11] Badr, R.O., Hosny, H.M., 2006, 'An HCI Pattern Language Management Tool', The 2006 International Conference on Computer Engineering and Systems, pp. 337-342.

[12] Jones, S.K., Roach, P. A., Perkins, S, 2008, 'Construction of Heuristics for a Search-Based Approach to Solving Sudoku', Research and Development in Intelligent Systems XXIV, pp 37-49.

[13] Lewis, R, 2007, 'MetaHeuristics Can Solve Sudoku Puzzles', Journal of Heuristics, Vol. 13, No. 4, pp. 387-401.

[14] Choi, D, Kim, H, Kim, J, 1999, 'Toward the construction of fun computer games: differences in the views of developers and players', Personal and Ubiquitous Computing, vol. 3, no. 3, pp 92-104.

[15] Simple Sudoku 2007, Simple Sudoku, Adelaide, Viewed 24 October 2008, < http://angusj.com/sudoku/>.

[16] Barr, P, Noble, J \& Biddle, R, 2007, 'Video game values: Human-computer interaction and games', Interacting with Computers, vol. 19, no. 2, pp 180-195.

[17] Sriboonruang, Y, Kumhom, P, Chamnongthai, K, 2006 'Visual Hand Gesture Interface for Computer Board Game Control', IEEE Tenth International Symposium on Consumer Electronics, pp. 1-6.

[18] Mason, S.G., Bohringer, R, Borisoff, J.F., Birch, G.E., 2004, 'Real Time Control of a Video Game with a Direct Brain-Computer Interface', Journal of Clinical Neurophysiology, pp. 404-408.

[19] Pineda, J.A., Silverman, D.S., Vankov, A, Hestenes, J, 2003, Learning To Control Brain Rhythms: Making a Brain-Computer Interface Possible, IEEE Transactions on Neural Systems and Rehabilitation Engineering, Vol. 11, No. 2, pp. 181-184. 